\title{
A Prolonged Response and Characteristics of Trabectedin Treatment of Metastatic Soft Tissue Sarcoma
}

\author{
Daniel Y. Reuben
}

\begin{abstract}
Unique features and treatment effects of trabectedin are presented in consideration of soft tissue sarcoma management. A prolonged time on trabectedin through 59 cycles is shown. This is one of the longer reported uses of trabectedin successfully to control disease. Adjunctive cytoreduction options with surgery, radiation or ablation are presented. Future studies would be helpful to investigate treatment holidays, the impact of multi-modality care and assessment of genetics of clonal metastases. This may assist in guiding and selecting patients for priority treatment with trabectedin.
\end{abstract}

Keywords: Trabectedin; Long; Duration; Soft; Sarcoma; Liposarcoma; Control; Chemotherapy

\section{Introduction}

The trabectedin chemotherapy experience is known for many years now and is an active agent for treatment of many soft tissue sarcomas. This agent does not have cumulative or generally increasing side-effects over time. Trabectedin may potentially remain effective for many months or years. These features are particularly noteworthy when counselling patients on trabectedin versus other systemic options. As predicting a response to therapy is currently difficult, it is ideal to remain on any satisfactory therapy to minimize the number of treatment changes and potential missteps and possible progression. On occasion excision, radiation or ablation of under-responding metastases can allow this. A number of investigators have now reported long treatment times with disease stability utilizing trabectedin [1-3]. This case report represents a patient with a prolonged treatment time on trabectedin. It also demonstrates the ability, and perhaps the importance of interfacing a multimodality treatment approach: incorporating surgical debulking and ablation of metastases for long term sarcoma control.

Manuscript submitted January 18, 2021, accepted January 25, 2021

Published online February 8, 2021

Division of Hematology and Oncology, Hollings Cancer Center, Medical University of South Carolina, MSC\# 635, 39 Sabin Street, Charleston, SC 29425, USA.Email: reubend@musc.edu

doi: https://doi.org/10.14740/jmc3655

\section{Case Report}

A 47-year-old woman presented with abdominal pains and hematuria in the year 2000. Imaging showed a left perinephric retroperitoneal mass. She underwent resection with left nephrectomy, left oophorectomy and partial colectomy for a well-differentiated liposarcoma. Clear margins were attained. In 2003 recurrence in multiple sites was seen within the abdomen. She underwent total abdominal hysterectomy and right oophorectomy, cholecystectomy and resection of retroperitoneal metastases with clear margins. In 2005 a second recurrence was noted in the region of the left flank requiring removal of recurrent liposarcoma from the left psoas and resection of small bowel with anastomosis and removal of retroperitoneal and peritoneal lesions. A third recurrence in 2011 necessitated resection of the right ureter, distal pancreatectomy, splenectomy and pelvic and retroperitoneal resections of tumor. Pathology review at this time showed both well-differentiated and de-differentiated liposarcoma. The patient experienced a partial small bowel obstruction as a consequence of her surgeries necessitating surgery for lysis of adhesions in 2014. In the subsequent year, she again had multifocal disease recurrence. Imaging showed a $4.7 \mathrm{~cm}$ mass at anterior abdominal cavity, a $2.6 \mathrm{~cm}$ mass at right lower quadrant inferior to the kidney, a 2.4 $\mathrm{cm}$ mid-abdominal wall lesion and other smaller lesions near the common bile duct, within mesentery and at left hemidiaphragm. The latter posed concern for herniation across the diaphragm. Three surgical opinions were sought and all recommended against resection with concern for margins, adjacent structures and short disease recurrence. She began systemic gemcitabine with a local oncologist. She tolerated three cycles but had disease progression. She was interested in second-line systemic therapy with trabectedin on a clinical trial prior to US FDA approval. At this time her computed tomographies (CTs) showed predominately a $2 \mathrm{~cm}$ left lung base mass at crus, and extensive peritoneal disease with a $1.9 \mathrm{~cm}$ subdiaphragmatic mass, a $3.3 \mathrm{~cm}$ infrarenal right lower quadrant mass, a $1.6 \mathrm{~cm}$ left peri-rectal mass, and a $5.8 \mathrm{~cm}$ anterior abdominal mass (Figs. 1, 2).

Trabectedin at $1.5 \mathrm{mg} / \mathrm{m}^{2}$ intravenous (IV) over $24 \mathrm{~h}$ continuous infusion every 21 days was administered in the outpatient setting with anti-emetics in early 2015 . Her first restaging showed stable disease, but by restaging after her fourth cycle, some mild response was seen. One year and 16 cycles later she had attained an approximate $20 \%$ regression of tumor measurements. At 2 years and 30 cycles her disease remained stable 


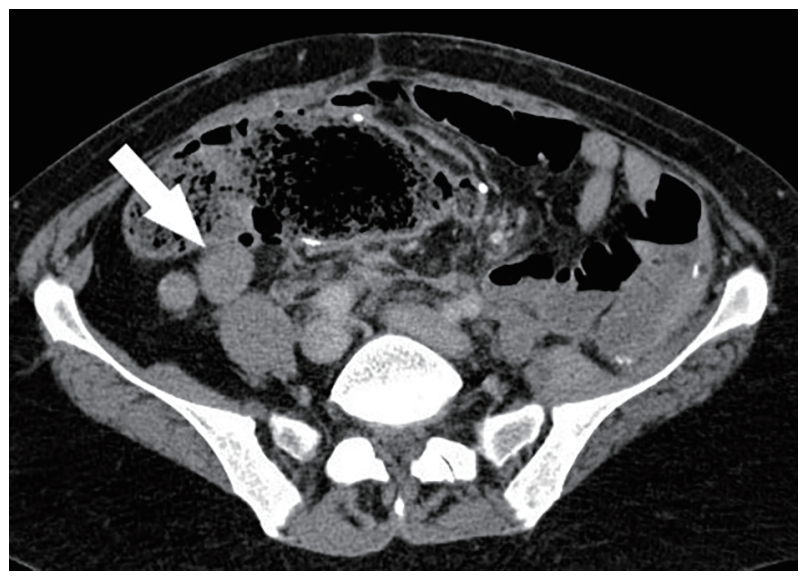

Figure 1. Computed tomography (CT) of abdomen/pelvis in 2015 prior to trabectedin with a representative abdominal liposarcoma metastasis (white arrow).

at previous measurements. Treatment was interrupted after 32 cycles for an episode of syncope and fall with ankle fracture necessitating surgical internal fixation and time for rehabilitation. Her symptoms were attributed to food poisoning and not chemotherapy, resulting in a 3-month delay in chemotherapy, but her disease remained stable. After 38 cycles, a mixed response was seen. Further regression was seen with regards to the left subdiaphragmatic mass now measuring $1.7 \mathrm{~cm}$ and a left peri-rectal nodule now $6 \mathrm{~mm}$. The anterior abdominal wall mass was stable at $4.2 \mathrm{~cm}$ (Fig. 3). The right infrarenal mass had grown to $3.9 \mathrm{~cm}$ however. A radiation oncology consult was elicited but with a single kidney radiation was felt too risky to attempt. Trabectedin was briefly continued. Further growth of the infra-renal metastasis to $4.7 \mathrm{~cm}$ occurred.

The patient returned to her previous surgeon who now agreed to excise the progressive metastatic tissue only. A dedifferentiated liposarcoma, grade 3 was seen with close margins. In April 2018, now post-op, she resumed trabectedin, cycle 41 . She continued for 6 months through cycle 49. She

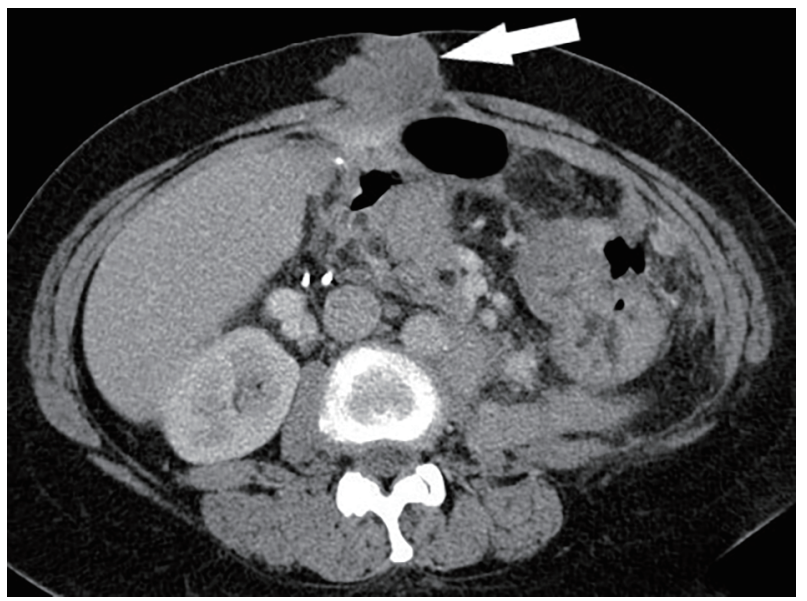

Figure 2. Computed tomography (CT) of abdomen/pelvis in 2015 prior to trabectedin with anterior abdominal wall peritoneal and soft-tissue metastasis (white arrow).

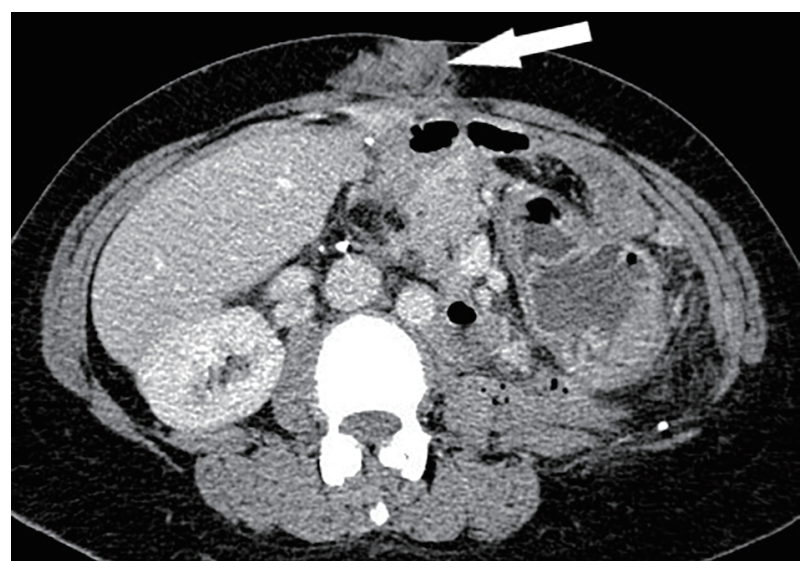

Figure 3. Computed tomography (CT) of abdomen/pelvis in 2017 demonstrating treatment effect with trabectedin. The anterior abdominal wall metastasis (white arrow) has decreased in size and stabilized.

failed to return for cycle 50 for a 6-month period. The patient was contacted multiple times and eventually returned. She stated she had a depressed episode. No new symptoms or exam findings were presented. Restaging showed continued stable low volume disease, and she resumed surveillance. By 9 months off treatment the patient had progression with the larger anterior abdominal wall mass measuring $4.6 \mathrm{~cm}$ (previously $2.6 \mathrm{~cm}$ ) and the newer abdominal metastasis now 2.8 $\mathrm{cm}$ (previously $1.5 \mathrm{~cm}$ ). Other areas remained unchanged. She again resumed trabectedin. Unfortunately these two metastatic sites continued to grow after another two cycles. Given dissatisfaction of this cosmetically, she was seen by surgical oncology at her current academic cancer center and undertook resection of the two anterior abdominal wall masses. Poorly differentiated sarcoma was noted on pathologic review. Margins were not completely cleared, but after two more cycles of chemotherapy, the area of resection and other metastatic sites continued to remain stable. A CT scan in the summer of 2020 showed stable findings except an enlarging right-sided (R-sided) abdominal wall mass of about $1 \mathrm{~cm}$ and a $2.2 \mathrm{~cm}$

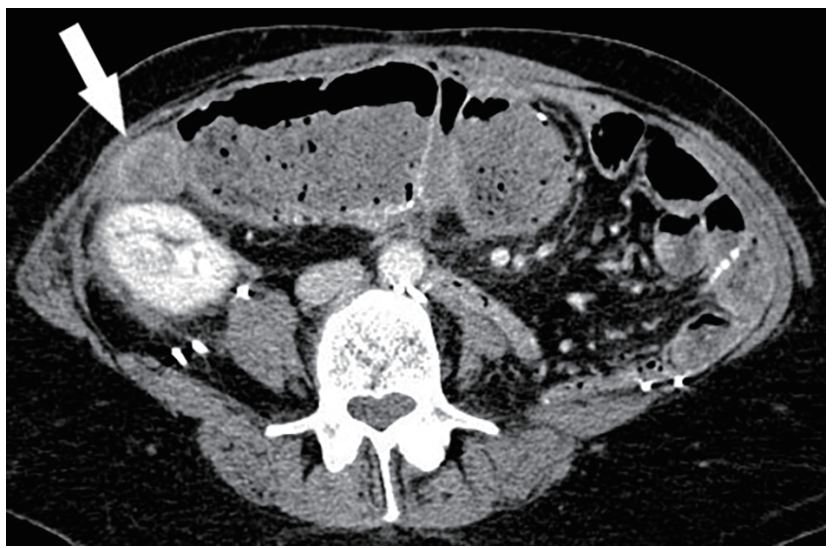

Figure 4. Computed tomography (CT) of abdomen/pelvis in 2020 revealed a new peritoneal metastasis despite trabectedin chemotherapy near a prior abdominal surgical bed. The lesion approximates bowel and right kidney (white arrow). 
R-sided intra-abdominal mass superior to the R kidney. The patient undertook her 59th cycle of trabectedin in July 2020. She again expressed interest in surgical excision of the growing lesions (Fig. 4). This was felt unlikely to be practical by surgery. She was evaluated by interventional radiology for an ablative attempt with cryotherapy. If it is successful and there are no other sites of progression, she could potentially resume trabectedin, cycle 60th.

\section{Discussion}

Trabectedin is an alkylating class of IV chemotherapy. It has been synthesized after investigation as an anti-cancer compound from ecteinascidin, a sea turbinate. The compound binds to the minor grove of DNA and it inhibits cell cycling at the G2/M phase of replication [4]. Whereas most chemotherapy agents create cumulative toxicity and the need for eventual dose-reduction, this is not generally seen with trabectedin. The case presented illustrates this. This patient did not ever require a dose-reduction. Of note there are three established dose levels for trabectedin $\left(1.5 \mathrm{mg} / \mathrm{m}^{2}, 1.2 \mathrm{mg} /\right.$ $\mathrm{m}^{2}, 1.0 \mathrm{mg} / \mathrm{m}^{2}$ ). Usually myelosuppression despite granulocyte colony-stimulating factor (G-CSF) dictates the need to adjust. Often this is seen early in treatment. In other cases, side-effects such as fatigue, transaminitis or myositis requires dose adjustments [5]. A lower dose does not seem to confer a less favorable ability to garner a treatment response. Here the patient tolerated treatment well with mild, cyclical cytopenias, fatigue and occasional myalgias but no chronic, escalating issues.

This patient undertook six cytoreductive surgeries along with an additional surgery to clear adhesions as a complication. While frequent surgery is not typical, it should be a consideration for some patients. What has been demonstrated is the ability to focus local strategies to eliminate non-responding metastases when a mixed response occurs. This can still provide continued systemic treatment harmoniously. Traditionally a mixed response begets many questions: Should therapy continue further if responding metastases outweigh an area of progression? Should the entire systemic therapy be changed regardless of prior benefits or convenience? How aggressive and how often should surgery be performed? Answers are not always clear. There are emerging data that repetitive surgical metastasectomy can lead to prolonged overall survival in this disease especially regarding lung metastases [6]. Outcome data to better delineate the benefits of external beam radiation, ablative interventional techniques and non-thoracic surgery is still needed.

The patient presented had treatment delays which were unanticipated. Usually a suspension of treatment for any reason will allow disease progression for patients with metastatic cancer. This patient had two interruptions with a 3-month holiday during ankle surgery and later a 9-month delay. This disease stability creates a hypothesis that arbitrary, perhaps short "off" windows, could be created for some patients [7]. An immediate patient-specific benefit of such is to reduce ongoing side-effects of chemotherapy or to mitigate certain risks. Improved pa- tient satisfaction and compliance may appear if interruptions to seemingly indefinite treatment can be shown to be appropriate. The creation of personal freedom and more independence from repetitive health care visits would be ideal. From a larger population standpoint, a reduction in resource use could potentially reduce costs. Developing a clinical trial to investigate this may be beneficial. As yet this maneuver of providing purposeful treatment holidays can't be recommended in standard practice.

Understanding the differences in growth characteristics and response of metastatic lesions to systemic therapy is elusive. Soft tissue sarcoma is often noted to metastasize to the lungs but hepatic and peritoneal metastases are also seen. Investigators have looked at gene expression to better understand this phenomenon. Recently over 5,000 genes have been assessed with 853 differentially expressed between the primary tumor and metastatic site in a mouse model. Certain signaling appears downregulated in metastatic sarcoma: epidermal growth factor receptor (EGFR), tumor necrosis factor- $\alpha$ (TNF- $\alpha$ ) and immune regulation. Signaling through wingless/ integrated (WNT), hypoxic mechanisms and metabolic processes were found to be upregulated. Focusing on candidate genes which may govern metastases is ongoing [8]. On the other hand, gene expression alone may not tell the tale. Epigenetic modulation has been seen to occur with respect to pulmonary metastases in sarcoma as well as other cancers [9]. Given the various permutation of cellular factors, not to mention extracellular domain issues, it will take some time to sort out the governing processes. Current clinical decision making still requires judgement in absence of prognostic signals for efficacy in treatment of most sarcomas.

\section{Conclusion}

The patient presented has both typical and atypical experiences in comparing with other patients with metastatic sarcoma. Typical features include the recurrences despite seemingly complete remissions and chemotherapy use. Surgical debulking, especially of lung metastases is a reasonable consideration. This can promote remissions, which as presented, lasted many years. An atypical feature shown relates to the high number of surgeries which is not always fostered or possible. The patient also had an unusually prolonged time on trabectedin chemotherapy without change in regimen. This patient was treated for 59 cycles of trabectedin which is one of the longer presentations known. No cumulative side-effects or toxicity was experienced. Trabectedin fulfills an additional tool for the medical oncologist in caring for these patients.

\section{Acknowledgments}

None to declare.

\section{Financial Disclosure}

None to declare. 


\section{Conflict of Interest}

There is no conflict of interest.

\section{Informed Consent}

Not applicable.

\section{Author Contributions}

Dr. Reuben has contributed solely and in whole to this report.

\section{Data Availability}

The author declares that data supporting the findings of this study are available within the article.

\section{References}

1. Rastogi S, Kalra K, Manasa P, Rajawat M, Mehta V. Long lasting response of trabectedin in patient with gastric leiomyosarcoma with liver metastasis: an update to previous report. Future Sci OA. 2019;6(1):FSO432.
2. Matsuda S, Tanaka K, Kawano M, Iwasaki T, Itonaga I, Tsumura H. Long-term disease control by trabectedin in a patient with dedifferentiated liposarcoma: A case report. Medicine (Baltimore). 2020;99(2):e18689.

3. Henry T, Fabre E, Baccar LS, Lamuraglia M. Longer survival in patients with metastatic uterine leiomyosarcoma treated with trabectedin: A case report. Mol Clin Oncol. 2019;10(3):387-390.

4. Erba E, Bergamaschi D, Bassano L, Damia G, Ronzoni S, Faircloth GT, D'Incalci M. Ecteinascidin-743 (ET-743), a natural marine compound, with a unique mechanism of action. Eur J Cancer. 2001;37(1):97-105.

5. http://www.janssenlabels.com/package-insert/productmonograph/prescribing-information/YONDELIS-pi.pdf.

6. Ceppa DP. Results of pulmonary resection: sarcoma and germ cell tumors. Thorac Surg Clin. 2016;26(1):49-54.

7. Pierantoni F, Maruzzo M, Brunello A, Chiusole B, Pusole G, Bezzon E, Basso U, et al. Trabectedin drug holiday and rechallenge in soft tissue sarcomas: report of 4 cases and literature review. Front Oncol. 2019;9:553.

8. Tang YJ, Huang J, Tsushima H, Ban GI, Zhang H, Oristian KM, Puviindran V, et al. Tracing tumor evolution in sarcoma reveals clonal origin of advanced metastasis. Cell Rep. 2019;28(11):2837-2850 e2835.

9. Reardon ES, Hong JA, Straughan DM, Azoury SC, Zhang M, Schrump DS. Pulmonary metastases exhibit epigenetic clonality: implications for precision cancer therapy. Ann Thorac Surg. 2015;100(5):1839-1848; discussion 1848. 